\title{
Nuclear analyses of solid breeder blanket options for DEMO: status, challenges and outlook
}

Pavel Pereslavtsev, Francisco A. Hernández, Guangming Zhou, Lei Lu, Christian Wegmann, Ulrich Fischer

\section{Objectives}

To support with neutronic analyses the systematic design development of the HCPB and alternative MLCB blankets

The following nuclear responses were assessed Tritium breeding ratio (TBR).

Effect of different design modifications on global TBR Power generation,

Power density distributions in materials,

Shielding performances of the DEMO

\section{Models}

I. Generic MCNP model

CAD model of DEMO baseline 2017

Full size $3 D$ model of $11,25^{\circ}$ torus DEMO segment

II. SMS blanket MCNP model

Roof shape FW $(20 \mathrm{~mm})$ with a W layer $(2 \mathrm{~mm})$

Faceted FW, empty breeder modules

III. Breeder module MCNP model

Heterogeneous FW (channels), BZ and BSS Hexagonal lattice of the breeder pins

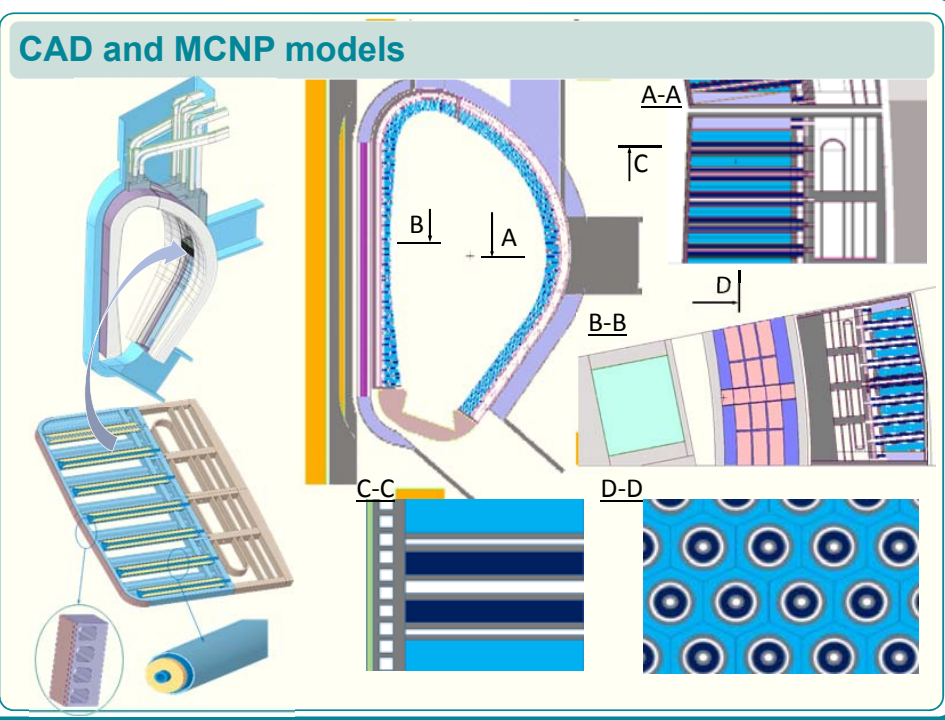

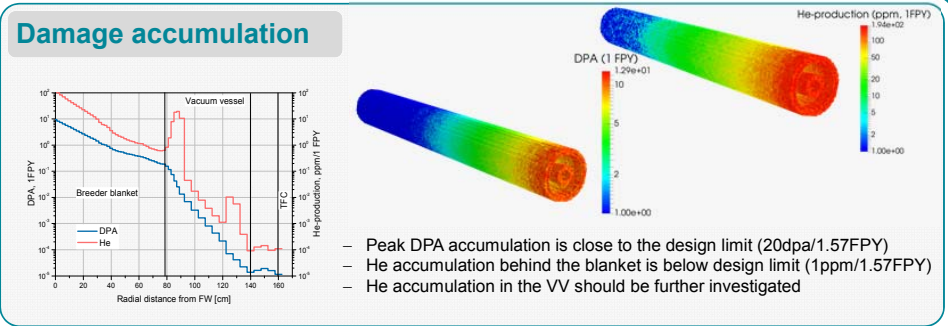

3D analyses (pin)
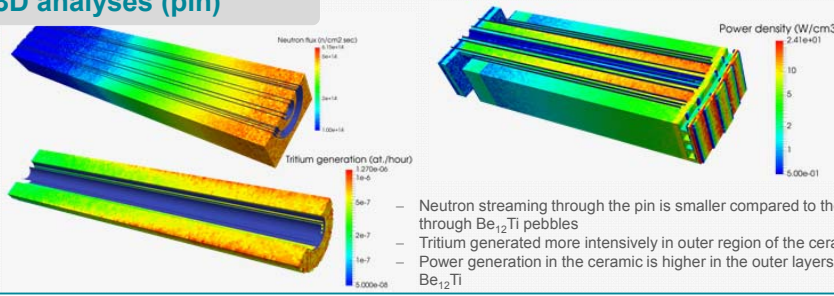

政
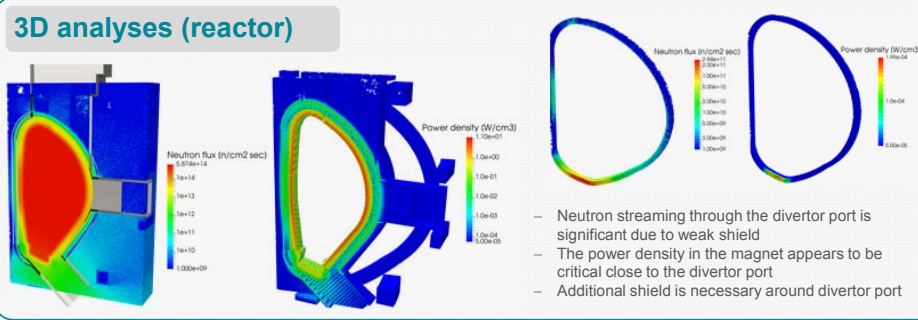

\section{Conclusions}

The innovative HCPB SMS blanket design based on the DEMO baseline 2017 was developed and successively optimized by means of coupled particle transport and thermal-hydraulic simulations

The new HCPB blanket provides sufficient TBR=1.16 and includes:

Breeder pins instead

ad of $\mathrm{Li}_{4} \mathrm{SiO}_{4}$

Alternative MLCB blanket design with $\mathrm{Pb}$ neutron multiplier was developed and optimized to provide TBR=1.13

The detailed heterogeneous modelling enables to assess a realistic tritium breeding 